\title{
Analisi ottica della turbolenza atmosferica
}

\author{
(Optical analysis of the atmospherical turbolence)
}

\author{
R. Giadea - D. Ventura
}

Ricevuto il 18 'Marzo 1972

\begin{abstract}
Riassunto. - Nella presente nota sono riportati alcuni fenomeni presentati da un fascio di raggi luminosi paralleli che si propagano negli strati bassi dell'atmosfera. In particolare si è osservata l'illuminazione da esso prodotta su uno schermo piano posto trasversalmente al fascio.

Per alcuni valori della distanza sorgente-schermo si è notata una distribuzione a struttura cellulare della intensità luminosa che si spostava con la velocità del vento. Allo scopo di giustificare questo fenomeno gli AA. hanno elaborato un modello di turbolenza atmosferica basato sull'ipotesi che nell'aria siano presenti discontinuità ottiche a simmetria sferica.

Si è potuto stabilire che una discontinuità di questo tipo provoca una focalizzazione del fascio ad una certa distanza con produzione di una pseudoimmagine della sorgente a forma di anello luminoso.

E stato cosi possibile spiegare l'aspetto della distribuzione dell'illuminamento osservato ed il fatto che la sovrapposizione degli effetti delle diverse bolle incontrate sia praticamente impercettibile.

Gli AA. concludono osservando che da questo esame è possibile analizzare la turbolenza, sia per quanto riguarda le dimensioni delle bolle, che per il valore della loro disomogeneità ottica.
\end{abstract}

SUmarr. - In the present paper the AA. reported some phenomena observed in a parallel light beam which propagated in the lower atmosphere. This beam was observed on a plane screen normally crossing the beam. For some source-screen distances a cellular intensity distribution was noted: this structure was moving with the wind speed.

In order to explain these phenomena the AA. suppose an atmospherical turbolence model based on the eddies with an optical inhomogeneity, which have a spherical symmetry. This inhomogeneity produce a beam focusing only at a give distance depending upon inhomogeneity characteristics.

The focusing forms a luminous ring which can be considered the source pseudoimage made by the eddies. 
The A A. show that the overlapping of the efrects by many eddies along the beam path is not important and conclude that same optical analysis can give the eddies dimension and the value of their optical inhomogeneities.

Nell'ambito dolle ricerche sulla turbolenza atmosferica eseguite in collaborazione con il Servizio Meteorologico dell'Aeronantica Militare e con l'Istituto Nazionale di Geofisica, sono state effettuate alcune osservazioni delle disomogeneità generate dalla turbolenza. Poiché queste disomogeneità non possono ovviamente raggiungere valori elevati, trattandosi di wn gas, l'aria, era necessario realizzare un sistema abbastanza sensibile alle variazioni dell'indice di rifrazione. Due potevano essere i metodi da seguire: o i metodi interferometrici ( $\left.{ }^{1}\right)$ o quelli rifrattivi (²). I primi sono però troppo sensibili, i secondi invece presentano degli indubbi vantaggi per la loro sensibilitì non eccessivamente spinta. Pertanto le nostre osservazioni sono state eseguite esaminando su uno schermo le sezioni di un fascio di luce, sufficientemente direzionale, a varie distanze dalla sorgente. Ise osservazioni sono state eseguite presso gli aeroporti di Fiumicino e di Pratica di Mare.

Le sorgenti arloperate sono state le seguenti:

1) proiettore catadiottrico con specchio di $50 \mathrm{~cm}$ di diametro con lampada a vapori di mereurio ad alta pressione da 200 watt; la brillanza della lampada era di 20.000 stilb con una dimensione di $3,25 \mathrm{~mm}^{2}$;

2) laser continuo ad IL-Ne della potenza di $3 \mathrm{mw}$ con apertura angolare variabile da 1 milliradiante a $10^{-2}$ milliradianti.

Ise sorgenti erano sistemate a distanze dal suolo variabili fra 1,5 metri e 8 metri ed il fascio di luce veniva fatto propagare in direzione pressocché parallela al terreno.

Dallesame del fascio su uno schermo posto a distanze diverse dalla sorgente, ¿ stato osservato il seguente fenomeno principale: l'illuminazione dello schermo si presentava con intensitì pressocché uniforme su tutta la sezione del fascio fino a distanze di 200-300 metri dalla sorgente; per distanze superiori e fino ad $1 \mathrm{~km}$ circa, sullo schermo cominciavano a delinearsi variazioni di intensita che sembravano viaggiare con la stessa velocità della componente del vento normale al fascio; per distanze ancora superiori le variazioni risultavano delineate più o meno nettamente assumendo talvolta un aspetto caratteristico. Sullo schermo si poteva così osservare una distribuzione dell'intensità a 
struttura cellulare, che si spostava con la velocità del vento. Le cellule, se isolate, erano pressocché circolari e se in gruppo e al limite a contatto l'una dell'altra, avevano una forma quasi esagonale. Le dimensioni di queste cellule, aventi i lati più luminosi del fondo, erano dell'ordine di 10-20 cm nella direzione verticale; le dimensioni orizzontali erano invece molto variabili e potevano presentarsi da una a tre volte quelle verticali. Nel caso di vento forte (> di 10 norli) le strutture sparivano dando luogo a delle strisce luminose orizzontali distanti circa $5-10 \mathrm{~cm}$. In alcuni casi poi tale struttura era clel tutto catica ell in altri, specie con velocità pressocché nulle del vento, spariva decisamente dando luogo a delle fluttuazioni di intensita apparentemente del tutto casuali.

E fuor di dubbio che le cause del fenomeno riscontrato siano da ricercarsi in una struttura discontinua dell'atmosfera, dovuta alla turbolenza su piccola scala. Gli eftetti clella turbolenza sulla propagazione della luce hanno richiamato l'attenzione degli stucliosi da molto tempo ed al riguarlo sono state elaborate molte teorie $\left({ }^{3}\right)$, basate, nella quasi totalità, sull'ipotesi che l'inclice di rifrazione presenti, punto per punto, delle variazioni del tutto casuali. Ma da queste premesse non si può arrivare a determinare che ma deviazione media del raggio ed uno spostamento medio della visuale geometrica in fumzione della distanza e dell'andamento delle fluttuazioni dell'indice di rifrazione.

E eviclente che per poter giustificare la struttura cellulare cla noi osservata, si debba invece partire da un altro punto di vista e cioè dall'ipotesi che nell'atmosfera esista non già una struttura del tutto casuale delle variazioni di indice di rifrazione, ma pinttosto una struttura a cellule ben definite. Nella presente nota abbiamo perciò elaborato un morlello teorico di disomogeneità che può spiegare il fenomeno da noi riscontrato: è ovvio che questo morlello per ora deve ritenersi una pura ipotesi di lavoro. Esso consiste nel supporre che nell'atmosfera, consiclerata otticamente omogenea, siano presenti delle cliscontinuità, aventi la forma di bolle sferiche, distribuite in molo pressocché uniforme nello spazio $\left(^{4}\right)$.

Dal punto di vista ottico l'indice di rifrazione $n$ potrà essere espresso la una funzione $n(r)$ tale che al centro di una generica bolla essa assuma il valore $n_{0}$, mentre ad una distanza $r$, generalmente minore della distanza media dei centri delle bolle, il suo valore sia $n_{\infty}>n_{0}$, essendo $n_{\infty}$ il valore caratteristico dell'inclice di rifrazione dell'atmosfera omogenea.

Trattanclosi di un modlello fenomenologico, non si entrerà nei particolari circa la formazione delle bolle, dovute probabilmente ad 
efTetti termici o a differente composizione; è bene però precisare che $\mathrm{i}$ valori dell'indice di rifrazione all'interno della bolla non possono assolutamente presentare delle discontinuita in quanto si tratta di bolle gassose le cui caratteristiche (temperatura, pressione e composizione) devono ovviamente raccordarsi con quelle presentate dallatmosfera omogenea, in quanto i processi di diffusione tendono ovviamente ad omogeneizzare tutta la massa d'aria.

Allo scopo di poter descrivere analiticamente l'andamento dell'indice di rifrazione nell'interno di una bolla, si è pensato ad una funzione del tipo

$$
n=a+b \cos \left(\pi 2^{-r^{v}}\right)
$$

dove $a$ e $b$ sono delle costanti, $r$ è la distanza del centro della bolla in unità arbitrarie e $v$ è un numero positivo maggiore della unità. Nella fig. 1 sono rappresentati gli andamenti di $n$ per $v=2$ e $v=4$; si ̀̀ scelto $\nu=4$, perché, pur non essenclo molto elevato, esso dà luogo ad una pendenza della curva a nostro giudizio più aderente ad una eventuale disomogeneità.

Poiché nella [1] si ha:

$$
\begin{array}{ll}
\text { per } r=\infty & n(\infty)=a+b=n_{\infty} \\
\text { per } r=0 & n(0)=a-b=n_{0} \\
\text { per } r=1 & n(1)=a
\end{array}
$$

l'unità normalizzata di $r$ risulta essere il raggio dello strato sferico per il quale l'indice di rifrazione assume il valore medio

$$
\boldsymbol{u}(1)=\boldsymbol{a}=\frac{n_{\infty}+n_{\mathrm{o}}}{2} .
$$

Calcoliamo ora la deviazione $\delta$ di un generico raggio luminoso che incontra una di queste bolle.

Si consideri un raggio incidente (fig. 2) che si propaga ad una distanza $\hbar$ dal raggio ad esso parallelo passante per il centro della bolla; il raggio subisce una deviazione $\delta$, che, per efietto della simmetria sferica, è data $\left(^{5}\right)$ da:

$$
\delta=2 \int_{n_{-2}}^{n^{*}} \frac{n h \mathrm{~d} n}{n \sqrt[1]{n^{2} r^{2}-n_{\infty}^{2} h^{2}}}
$$

dove $n^{*}$ ̀̀ il valore dell'indice di rifrazione dello strato sferico di raggio $r^{*}$, che rappresenta la minima distanza della traiettoria del raggio 
luminoso dal centro della bolla; ovviamente il raggio risulterà tangente alla sfera di raggio $r^{*}$. Il calcolo dello integrale [2] è abbastanza laborioso; pertanto esso è stato eseguito per via numerica presso il Centro

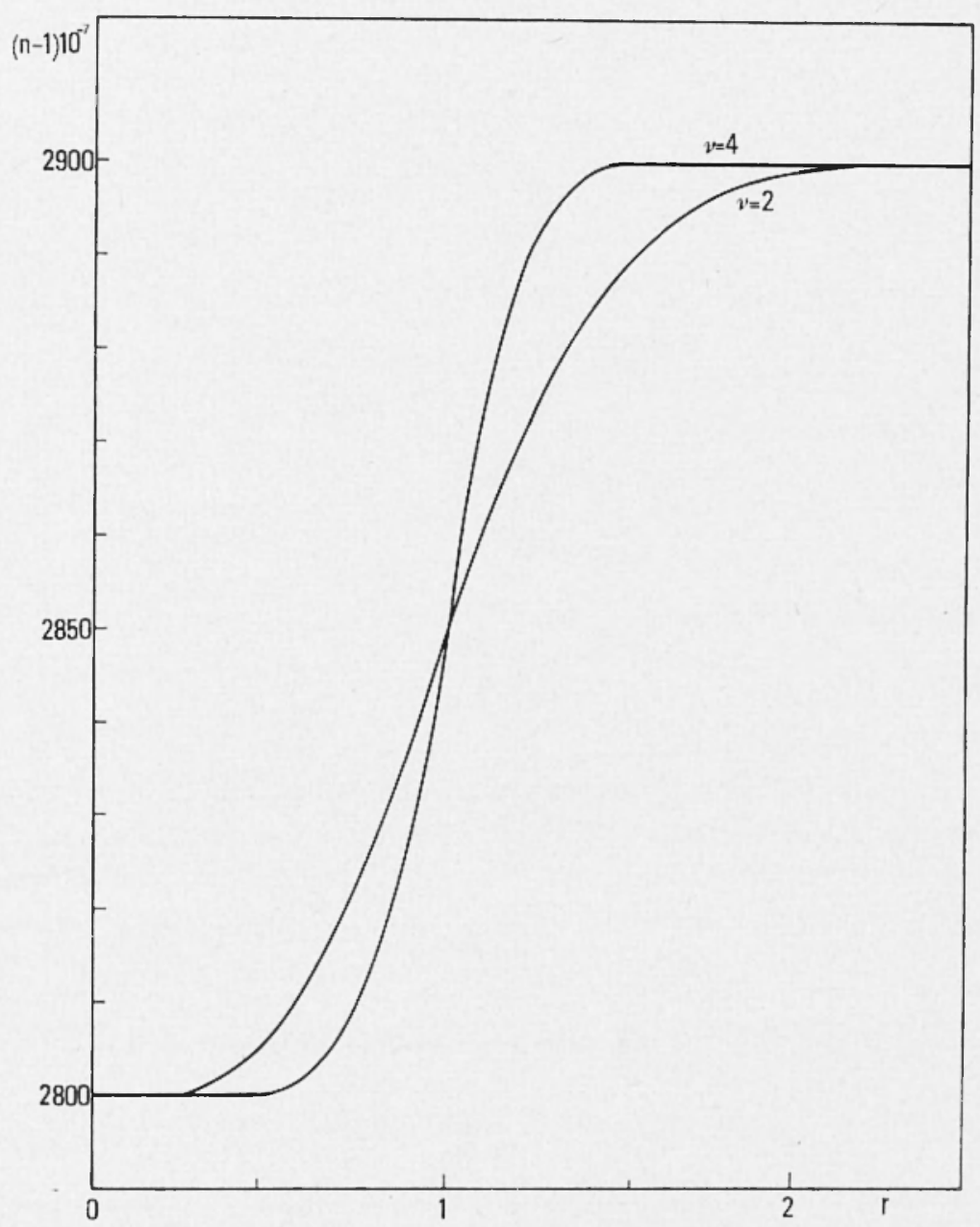

Fig. 1

di Calcolo dell'Università di Roma (Univac 1108). Nella fig. 3 è riportato un andamento tipico di $\delta$ in funzione di $h$ per $b=5 \cdot 10^{-6}$.

Nella ipotesi che nella stessa atmosfera possano esistere disomogeneità sferiche più o meno intense, sono stati calcolati $\mathrm{i}$ valori di 
$\delta$ corrispondenti a cliversi valori di $b$, ma tenendo costante $n_{\infty}=a+b=1,00029$.

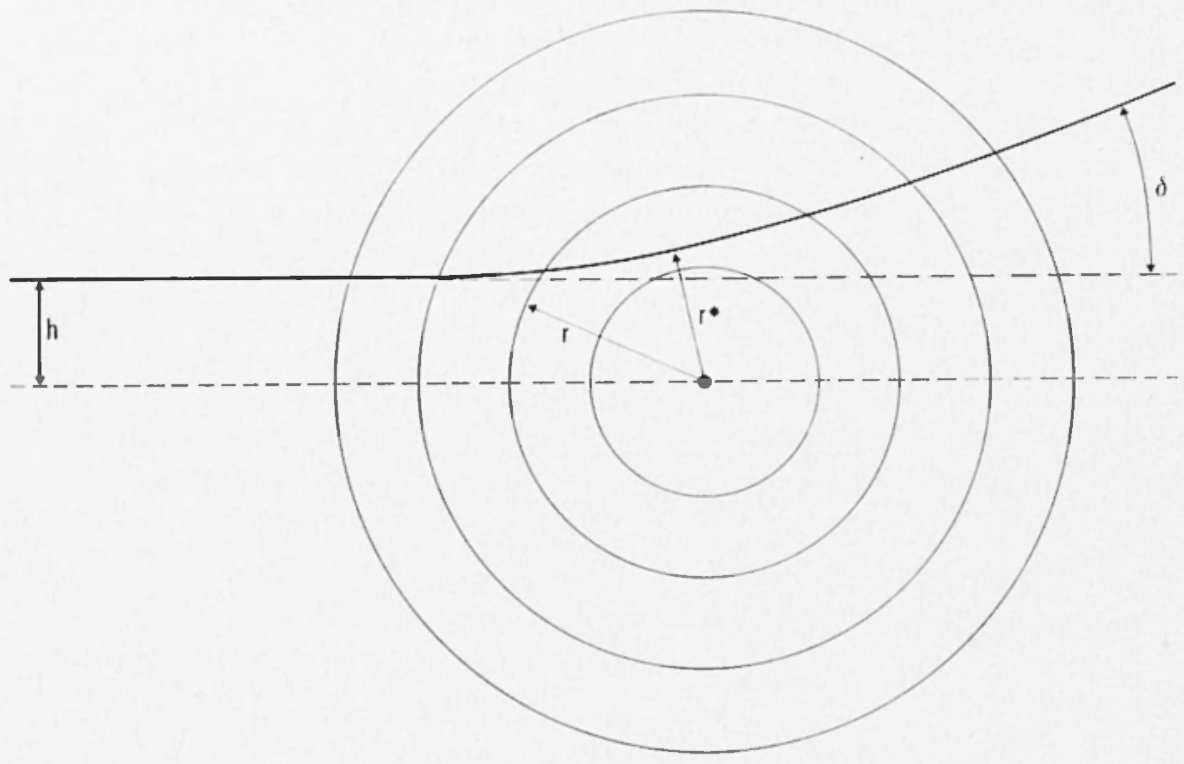

Fig. 2

I risultati del calcolo sono riassunti nella Tabella 1 dove sono stati riportati aleuni valori di $\delta$ corrispondenti alla deviazione massima ed il corrispondente valore di $h$ per alcuni valori di $b$. Nell'ultima coloma sono stati riportati i valori di $\left(\delta_{\text {max }}\right)_{\text {ealc }}$, secondo la relazione

$$
\delta_{\max }=6,096
$$

in quanto si verle che essi sono praticamente proporzionali a $b$.

Tabella 1

\begin{tabular}{|c|c|c|c|}
\hline$b$ & $h$ & $\delta_{\max }$ & $\left(\delta_{\max }\right)_{\text {calc }}$ \\
\hline $10^{-7}$ & 0,9 & $6,086.10^{-7}$ & \\
$5.10^{-7}$ & 0,9 & $3,043.10^{-6}$ & $6,09.10^{-7}$ \\
$10^{-6}$ & 0,9 & $6,086.10^{-6}$ & $3,05 \cdot 10^{-6}$ \\
$5.10^{-6}$ & 0,9 & $3,043.10^{-5}$ & $6,09.10^{-6}$ \\
$10^{-5}$ & 0,9 & $6,087.10^{-5}$ & $3,05.10^{-5}$ \\
& & & $6,09.10^{-5}$ \\
\hline
\end{tabular}


ì possibile adesso verificare che l'andamento di $\delta$ può portare ad una focalizzazione del fascio luminoso: infatti i valori di $\delta$, inizialmente

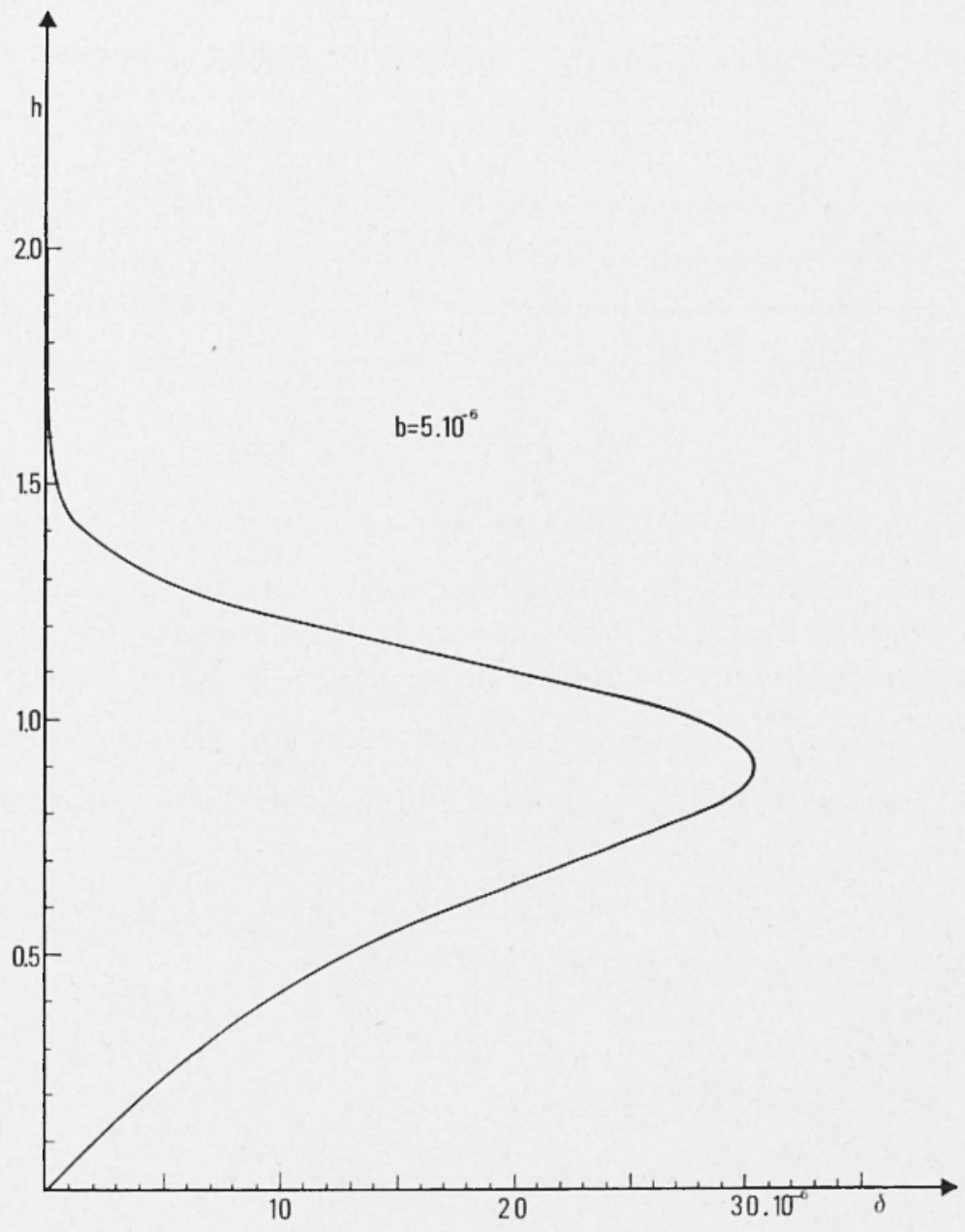

Fig. 3

crescenti all'aumentare di $h$, raggiungono un massimo e successivamente decrescono tendendo a zero (fig. 4); la diminuzione di $\delta$ per valori crescenti di $h$ comporta la focalizzazione dei raggi luminosi ad una distanza $D$ tale che (fig. 5)

$$
h+D \delta=\text { cost. }
$$


rioi:

$$
D=-\frac{\mathrm{d} h}{\mathrm{~d} \delta}
$$

Ma all'aumentare di $h, \frac{\mathrm{d} \delta}{\mathrm{d} h}$, inizialmente positivo, decresce fino

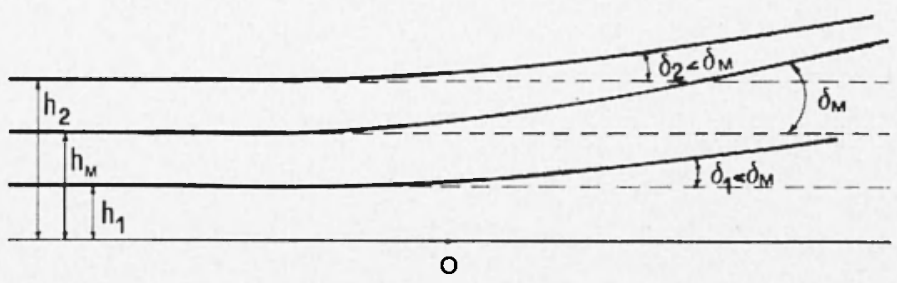

Fig. 4

a cambiare di segno arrivando ad un valore minimo $\left(\frac{\mathrm{d} o}{\mathrm{~d} h}\right)_{\mathrm{c}}$; successivamente aumenta e si annulla quando $h=\infty$ (fig. 6); quindi per $h_{c}<h<\infty$ i raggi focalizzeranno a varie distanze. Da una analisi sommaria della figura si vele che per un tratto, compreso tra i valori di $h_{1}$ e $h_{2}$ la $\frac{d \delta}{\bar{a} h}$ si mantiene pressocché costante: ciò significa che i raggi incidenti sulle bolle con distanze iniziali dall'asse di simmetria

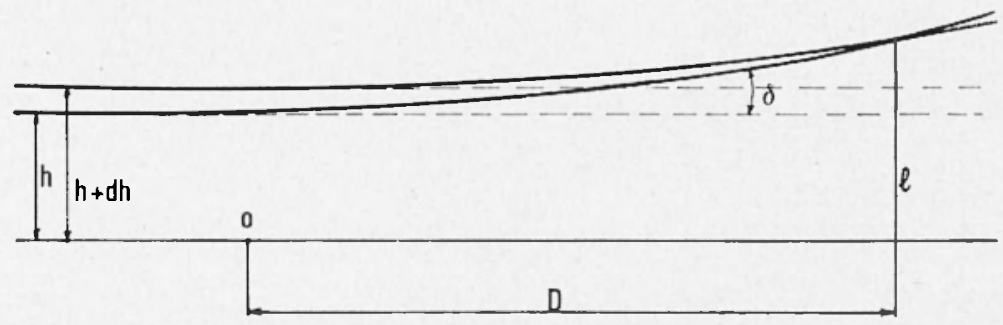

Fig. 5

comprese tra $h_{1}$ e $h_{2}$ focalizzeranno ad una stessa distanza $D_{c}=-\left(\frac{\mathrm{d} h}{\mathrm{~d} \delta}\right)$. I'interpretazione più plausibile di tutto ciò è che ogni bolla produca, per ogni sorgente puntiforme, una pseudoimmagine a forma di anello alla distanza $D_{c}$ il cui raggio è dato da (fig. 5)

$$
R^{\prime}=h_{c}+D_{c} \delta_{c}=h_{c}-\delta_{c}\left(\frac{\mathrm{d} h}{\mathrm{~d} \delta}\right)_{c} .
$$


Questi anelli tendono a sovrapporsi e quindi formeranno delle concentrazioni di luce, che, data la simmetria del fenomeno e ammessa l'uniformitì di distribuzione delle bolle, tendono a formare i lati degli esagoni osservati.

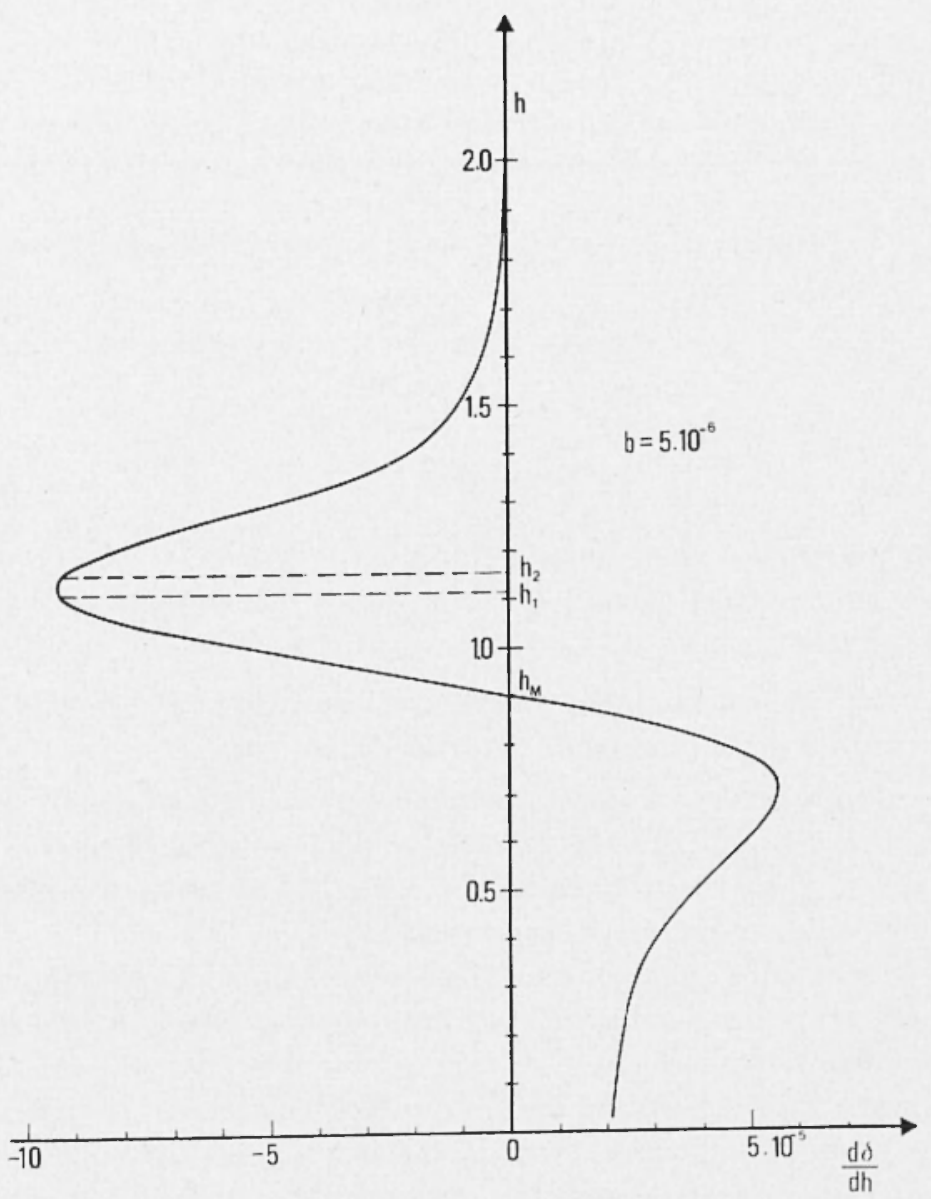

Fig. 6

Nella tabella 2 sono riportati i valori di $\left(\frac{d w}{d h}\right)_{c}$, i corrispondenti valori di $h_{c}$ e di $\delta_{c}$, le distanze di focalizzazione calcolate $D_{c}$ e gli intervalli $\Delta h$ per i quali si ha una focalizzazione in un intervallo $\frac{\Delta D_{c}}{D_{c}}$ pari al $5 \%$. 
Tabclla 2

\begin{tabular}{|r|c|c|c|c|c|}
\hline$b$ & $h_{c}$ & $\delta_{c}$ & $(\mathrm{~d} \delta / \mathrm{d} h)_{c}$ & $D_{c}$ & $\Delta h=\eta$ \\
\hline $10^{-7}$ & 1,13 & $3,512.10^{-7}$ & $1,865.10^{-6}$ & $5,36.10^{5}$ & 0,08 \\
$5.10^{-7}$ & 1,13 & $1,756.10^{-6}$ & $9,320.10^{-6}$ & $1,07.10^{4}$ & 0,08 \\
$10^{-6}$ & 1,13 & $3,512.10^{-6}$ & $1,865.10^{-5}$ & $5,36.10^{4}$ & 0,08 \\
$5.10^{-6}$ & 1,13 & $1,756.10^{-5}$ & $9,320.10^{-5}$ & $1,07.10^{4}$ & 0,08 \\
$10^{-5}$ & 1,13 & $3,512.10^{-5}$ & $1,865.10^{-4}$ & $5,36.10^{3}$ & 0,08 \\
\hline
\end{tabular}

Gli andamenti di $\delta_{c}, D_{c}$ ed $R^{\prime}$ sono approssimati abbastanza bene dalle seguenti funzioni:

$$
\begin{aligned}
\delta_{c} & =3,51 b \\
D_{c} & =\frac{5,36 \cdot 10^{-2}}{b} \\
R^{\prime} & =1,31
\end{aligned}
$$

È necessario tener presente che mentre la focalizzazione può presentare valori anche elevati, le deviazioni sono relativamente piccole. Questo significa che:

1) le strisce luminose hanno praticamente la stessa dimensione dello strato avente l'indice di rifrazione medio $a$;

2) i raggi provenienti da bolle uguali si focalizzano ad una stessa distanza; quindi sullo schermo si possono osservare solo le concentrazioni di luce provenienti dalle bolle che si trovano ad una ben determinata distanza dallo schermo stesso. Questa è la ragione per la quale la struttura cellulare non viene cancellata dalla sovrapposizione di queste concentrazioni dovute alle bolle che inevitabilmente si trovano sul percorso della luce.

Infatti sullo schermo si possono vedere solo gli effetti delle discontinuità poste ad una certa distanza $D$ e non le più vicine né le più lontane, come se esistesse solo uno strato perturbatore alla distanza $D$.

Si potrebbe però pensare che i successivi passaggi attraverso le molte discontinuità, attraversate da un generico raggio di luce prima di arrivare sullo schermo, possano cancellare con le successive deviazioni ogni traccia di struttura cellulare. Ma, come vedremo tra poco, questo efletto è abbastanza piccolo. Infatti di tutto il fascio di luce attraversante una generica bolla interessa solo il piccolo strato compreso tra le altezze $h_{1}$ e $h_{2}$ (lig. 6), in quanto solo questo andrà a focalizzarsi sullo schermo. Orbene, nel suo cammino questa sezione potrà incontrare 
una generica bolla in una di queste due posizioni (verli figg. $\tau$ e 8 ); cioè se $s$ è la distanza lungo la normale al fascio tra il centro del fascio e quello della bolla ed $\eta$ lo spessore $\Delta h$ del fascio e della bolla, si potrà avere

$$
\begin{array}{ccc}
0 & \eta \leqslant s<2 & \text { fig. } 7 \\
0 & s \leqslant \eta & \text { fig. } 8 .
\end{array}
$$

Se $s$ è minore di $\eta$ ciò significa che fascio e strato convergente della bolla sono praticamente sovrapposti. $\dot{\mathrm{i}}$ ovvio che tutte le lunghezze sono normalizzate al raggio dello strato per il quale l'indice assume il suo valore medio; il valore 2 che appare nella prima disuguaglianza rappresenta il raggio della bolla, in quanto la differenza $n(\infty)-n(2)$ è praticamente nulla: si ha infatti dalla [1] per $v-4$

$$
n(\infty)-n(2)=2,4 \cdot 10^{-5} b .
$$

Nel primo caso (fig. 7) la maggior parte del fascio incide sulla bolla in zone dove la conseguente deviazione è praticamente costante

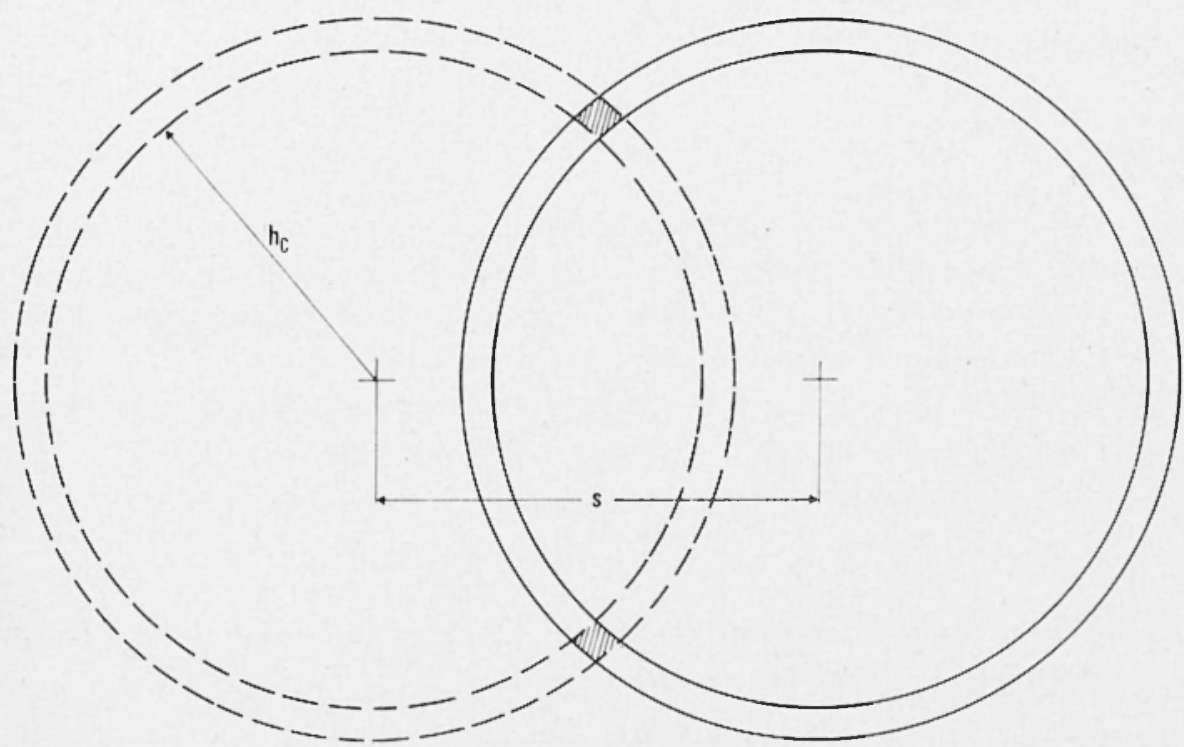

Fig. 7

per tutta la sezione del fascio (rappresentata a tratti nel disegno) ed una piccola parte inciderà sulla zona di ampiezza $\Delta h$ (v. tab. 2) dove la focalizzazione molto forte porta ad una dispersione del fascio. La prima 
non darà luogo che a delle deviazioni pressocché uguali per tutta la sezione del fascio che in totale tenderanno a compensarsi producendo sullo schermo delle fluttuazioni della forma della pseurloimmagine, del resto ben avvertibili nelle nostre osservazioni. La seconda invece tenderà a disperdere il fascio e quindi a clistruggere completamente la forma di questa pseurloimmagine e di conseguenza a cancellare ogni traccia di struttura cellulare.

Cerchiamo di valutare ora questo efletto: si consirleri l'atmosfera riempita di bolle sferiche con i centri posti a rlistanza $d$; la probabilità che ci sia una qualsiasi sovrapposizione di due bolle di raggio $r=2$ sarà:

$$
p_{1}=\frac{\pi(4)^{2}}{\pi d^{2}}=\frac{16}{d^{2}} .
$$

Ora per ogni bolla incontrata in questa conclizione (v. fig. 7 ) solo una porzione $2 \eta$ - della sezione del fascio viene completamente deviata e quindi, affinché non ci sia più traccia rlella struttura cellulare, occorrerà che tutte le porzioni della sezione del fascio incontrino una bolla; poiché ad ogni incontro viene eliminata un'area $2 \eta^{2}$, occorreranno $N$ incontri per poterla eliminare tutta, flove $N$ è dato da

$$
N=\frac{2 \pi h_{c} \eta}{2 \eta^{2}}=-\frac{h_{c}}{\eta}
$$

Ma non tutti gli incontri saranno utili alla cancellazione efiettiva; infatti se pensiamo di surldivirlere la sezione del fascio in $N$ parti, la probabilità che al primo incontro sia cancellata un'area $2 \eta^{2}$ è 1 , cioè la certezza; per un secondo incontro la probabilità diventa $(N-1) / N$ per un terzo $(N-2) / N$ e cosi via. Quincli la probabilità che tutte le porzioni della sezione siano cancellate sarà data da

$$
p_{2}=\frac{N !}{N^{N}}
$$

e, nell'ipotesi che $N$ sia sufficientemente grancle, si può usare la formula approssimata di Sterling, ottenenrlo

$$
p_{2}=e^{-N} \sqrt{2 \pi N}
$$

Per la cancellazione totale occorreramno quindi $N_{e}$ incontri effettivi dati da

$$
N_{e}=\frac{N}{p_{1} p_{2}}=\left(\frac{d}{4}\right)^{2} \sqrt{\frac{h_{c}}{2 \eta}} e^{\pi \frac{h_{c}}{\eta}}
$$


ed il fascio di luce dovrà percorrere un tratto $L$ dato da

$$
L=N_{e} d=\frac{d^{3}}{16} \sqrt{\frac{h_{c}}{2 \eta}} e^{\pi \frac{h_{c}}{\eta}} .
$$

Si tratta quindi di un percorso enorme: infatti basta porre per esempio $d=4$ (bolle a contatto), $h_{c}=1,13, \eta=0,08$, ponendo l'unità di normalizzazione $r=1=10 \mathrm{~cm}$, per trovare

$$
L=1,03 \cdot 10^{14} \mathrm{~km} \text {. }
$$

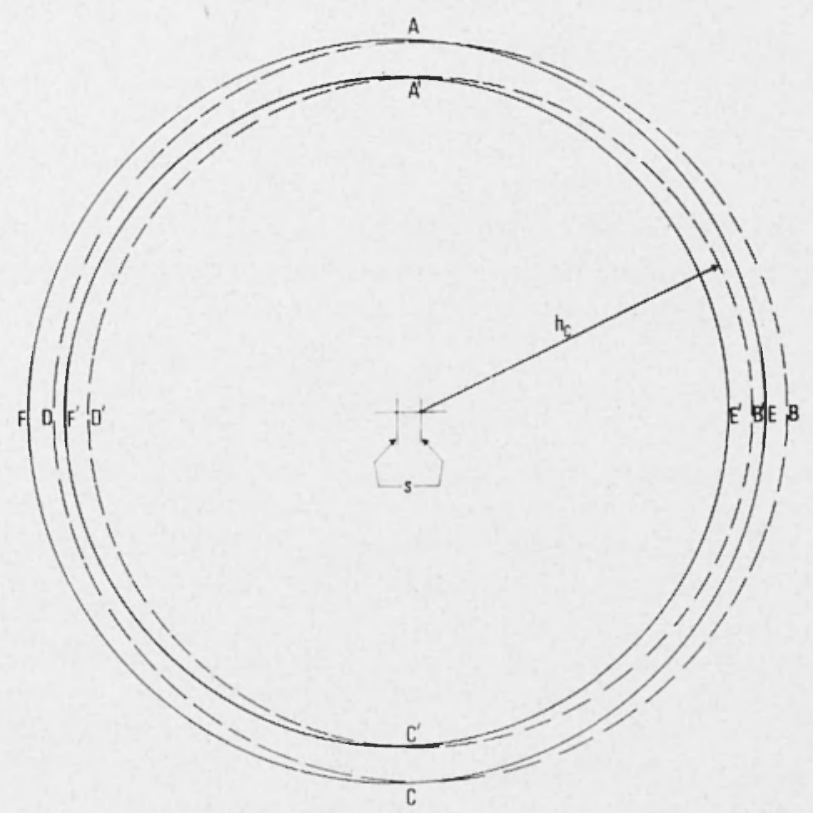

Fig. 8

Passiamo ora al caso di fig. 8; sia $A B C D A$ il contorno esterno del fascio di luce e $A^{\prime} B^{\prime} C^{\prime} D^{\prime} A^{\prime}$ quello interno. Siano inoltre $A E C F A$ e $A^{\prime} E^{\prime} C^{\prime} F^{\prime} A^{\prime}$ i contorni della zona, nel disegno tratteggiata, compresa tra $h_{1}$ e $h_{2}$ della generica bolla incontrata dal fascio. Nella ipotesi ammessa solo la porzione compresa tra $A E C D A$ e $A^{\prime} B^{\prime} C^{\prime} F^{\prime} A^{\prime}$ sarà deviata completamente. L'area restante è formata dalle due lunule $A B C E A$ e $A^{\prime} D^{\prime} C^{\prime} F^{\prime} A^{\prime}$, le cui superfici sono date da $\frac{\pi}{2} h_{c s}$ e $\frac{\pi}{2}\left(h_{c}-\eta\right) s$. Quindi 
di tutto il fascio rimane inalterata solo l'area

$$
\frac{\pi}{2}\left(2 h_{c}-\eta\right) s
$$

e poiché l'area della sezione del fascio è $2 \pi h_{c} \eta$, ciò significa che la sezione del fascio utile alla formazione della pseudoimmagine sullo schermo ì ridlotta arl una frazione $\mu$, rata da

$$
\mu-\frac{2 h_{c}-\eta}{4 h_{c}} \cdot \frac{s}{\eta}=\frac{s}{2 \eta} .
$$

Ia probabilità che il fascio sia ridlotto ad una frazione minore o tutt'al più uguale a $\mu$ è allora clata da

$$
p_{3}=\frac{\pi s^{2}}{\pi d^{2}}=\left(\frac{2 \eta}{d}\right)^{2} \cdot \mu^{2}
$$

in quanto solo se i centri si trovano ad una distanza minore di s la sezione del fascio viene ridotta alla frazione $\mu$. Pertanto il fascio dovrà percorrere un tratto

$$
L=\frac{d}{p_{1} p_{3}}-\frac{d^{5}}{64 \eta^{2}} \frac{1}{\mu^{2}}
$$

e nel nostro esempio

$$
L=\frac{250}{\mu^{2}} \mathrm{~m} .
$$

Se poniamo come limite di visibilità della struttura $\mu=0,1$, avremo

$$
L=2 \check{\mathrm{km}}
$$

ciò̀ il fascio dovrebbe percorrere venticinque chilometri per potersi ridurre ad 1/10 e quindi per poter perdere la sua primitiva struttura.

Pertanto, poiché dalle nostre osservazioni si è visto che le distanze di focalizzazione sono dell'ordine di uno-rlue chilometri, si puó arguire che a queste distanze la focalizzazione non viene praticamente perturbata dai successivi passaggi attraverso le bolle.

Da tutte queste considerazioni si può concludere che sia accettabile l'ipotesi di lavoro di partenza, cioè l'ipotesi di una turbolenza a struttura cellulare. Se si ammette questo morlello, il metorlo da noi usato può essere ritenuto arlatto per una valutazione della turbolenza: infatti 
dalle dimensioni delle cellule osservate sullo schermo è possibile ricavare le dimensioni reali dlelle bolle, mentre rlalla distanza minima alla quale sono visibili le cellule stesse si può ricavare $b$, valore legato alla disomogeneità ottica presentata dalla bolla; infatti si ha $\Delta n=2 b$.

Siamo lieti di aver l'occasione di ringraziare il Dott. F. Pellegrini del Servizio Meteorologico dell'Aeronautica per la partecipazione al progetto nella sua prima fase ed il Sig. Antonino Sorce del Centro di Calcolo dell'Universita di Roma per l'assistenza durante l'elaborazione rei calcoli.

\section{BIBLIOGRAFIA}

(1) Tra i vari lavori sperimentali si citano:

Burlamaccir P., Consortini A., Roncin L., 1967. - Applied Optics, 6, p. 1273.

Bertolotti M., Carnevale M., Muzi L., Sette D., 1968. - Applied Optics, 7, p. 2246.

Bouricius G. M. B., Clifford S. F., 1970. - "J.O.S.A.", 60, p. 1484. Bertolotti M., Muzis L., Sette D., 1970. - "J.O.S.A.", 60, p. 1603.

(2) Tra i vari lavori sperimentali si citano:

Вескмann P., 1965. - Radio Science, 69 D, p. 629.

Burlamaccir P., Consortini A., 1967. - "Optical Acta ", 14, p. 7.

Fried D. L., Mevers G. E., Keister J1, M.P., 1967. - "J.O.S.A.", 57, p. 787.

$\left.{ }^{3}\right)$ Tra i vari lavori teorici si citano:

TAtarski V. I., 1961. - Wave propagation in a turbulent medium, MeGrawHill, New York.

Consortini A., Roncir L., 1972. - Applied Optics, 11, p. 1205.

(4) Sutton 0. G., 1953. - Micrometeorology, McGraw-Hill, New York.

Scorer R. S., Ludlam F. II., 1953. - "J. Roy. Met. Soc. ", 79, p. 94. FeA G., 1962. - Proc. of the Symposium on solar seeing. C.N.R., Roma, p. 111 .

Symposium N. 19 de l'Union Astronomique Internationale, 1963. "Bull. Astron. de l'Observ. de Paris", XXIV, fasc. 2 e 3.

(5) Bounsse II., 1926. - Optique Géométrique Supérieure, Delagrave, Paris. 